\title{
Simulation of a DI Diesel Engine Performance Fuelled on Biodiesel Using a Semi-Empirical 0D Model
}

\author{
Claude Valery Ngayihi Abbe ${ }^{1,2}$, Robert Nzengwa ${ }^{1,2}$, Raidandi Danwe', \\ Zacharie Merlin Ayissi ${ }^{2}$, Marcel Obounou ${ }^{3}$ \\ ${ }^{1}$ Laboratory of Applied Mathematics, National Advanced School of Engineering, University of Yaoundé, Yaoundé, Cameroon \\ ${ }^{2}$ Faculty of Industrial Engineering, University of Douala, Douala, Cameroon \\ ${ }^{3}$ Department of Physics, Faculty of Sciences, University of Yaoundé, Yaoundé, Cameroon \\ Email: ngayihiclaude@yahoo.fr
}

Received October 17, 2013; revised November 17, 2013; accepted November 24, 2013

Copyright (C) 2013 Claude Valery Ngayihi Abbe et al. This is an open access article distributed under the Creative Commons Attribution License, which permits unrestricted use, distribution, and reproduction in any medium, provided the original work is properly cited. In accordance of the Creative Commons Attribution License all Copyrights (C) 2013 are reserved for SCIRP and the owner of the intellectual property Claude Valery Ngayihi Abbe et al. All Copyright (C) 2013 are guarded by law and by SCIRP as a guardian.

\begin{abstract}
Diesel engines have proven over the years important in terms of efficiency and fuel consumption to power generation ratio. Many research works show the potential of biodiesel as a substitute for conventional gasoil. Mainly, previous and recent researches have focused on experimental investigation of diesel engine performance fuelled by biodiesel. Researches on the mathematical description of diesel engine process running on biodiesel are scarce, and mostly about chemical and thermodynamic description of the combustion process of biodiesel rather than performance studies. This work describes a numerical investigation on the performance analysis of a diesel engine fuelled by palm oil biodiesel. The numerical investigation was made using a semi empirical 0D model based on Wiebe's and Watson's model which was implemented via the open access numerical calculation software Scilab. The model was validated first by comparing with experimental pressure and performance data of a one cylinder engine at rated speed and secondly by comparing with a six cylinders engine performance data at various crankshaft rotational speeds. Simulations were then made to analyze the engine performance when running on biodiesel. The calculations were made at constant combustion duration and constant coefficient of excess air. Results showed that the model matches the overall experimental data, such as the power output and peak cylinder pressure. The ignition delay was somehow underestimated by the model for the first experiment, which caused a slight gap on in cylinder pressure curve, whereas it predicted the average ignition delay fairly well for the second set of validation. The simulations of engine performance when running on biodiesel confirmed results obtained in previous experimental researches on biodiesel. The model will be further investigated for engine control when shifting to biodiesel fuel.
\end{abstract}

Keywords: Semi-Empirical 0D Model; Scilab; Biodiesel; Performance; Diesel Engine

\section{Introduction}

Reaching lower toxicity of exhaust gases, whilst reducing fuel consumption is one of the modern trends in automobile engineering research. The characteristics of fuel play an important role in the performances of the engine. Gasoil has been for years the main fuel for diesel engine but, due to the reducing availability of fossil energy resources and the stricter rules on engine emission [1], and researchers have been investigating the use of alternative fuels. Biodiesel, which is obtained through transesterification process of vegetable oil, has been proven to be a high potential substitute for conventional gasoil [1-3].
Simulation and mathematical modeling of diesel engine are scientific topics carried out by several research works. Nowadays, there mainly exist three types of approaches for diesel engine simulation: 0 dimensional thermodynamic, quasi dimensional and multi dimensional (Computational Fluid Dynamic). A well documented discussion on these models can be found in [4].

Many commercial computer codes based on computational fluid dynamic (CFD) [5] provide useful tools to simulate in diesel engine processes. CFD permits simulating real engine conditions with the aim of understanding how physical and chemical conditions will affect engine performances. One of the main disadvantages of 
CFD tools is that they are too slow to be implemented in car vehicles control software and their use as optimization or simulation tools which have a very high computer cost. Car control software mostly uses semi empirical 0D model due to their simplify approach, their quickness of implementation inside the electronic unit control of the engine. For engine simulation and optimization, several commercial codes using semi empirical models exist [6, 7]. The cost for license acquisition and the access to source codes are some of the limitations of this software in term of scientific research. To get away from these limitations we used an open source numerical calculation software called Scilab [8] in which we implemented our model.

The model we implemented in our study is based on the engine performance Wiebe model [9]. It has been for years used in diesel engine simulation, and it requires adjustable experimental coefficients related to engine specification. Since the scope of the work is about defining effect of biodiesel on engine performances, the experimental coefficient can be considered constant since they don't depend on the fuel used.

One of the limitations of the Wiebe model is that it doesn't predict well the combustion during the premix phase of combustion. That limitation is overcome by Watson model of heat release in diesel engine $[4,10]$. Both Wiebe and Watson model don't take into account the ignition delay of the injected fuel in the cylinder, thus obliging us to find a suitable relationship to predict ignition delay. Pischinger et al. [11] defined a correlation between the length of the fuel spray at which ignition occurs and the temperature and pressure at the start of injection angle. Others strong relationship between ignition delay and start of injection angle was experimentally derived by Razlejtsev [12] and Lechivskii [13]. In these relationships, the ignition delay is described using an Arrenhius correlation between the pressure and temperature at start of injection angle and activation energy of the fuel. However, these relationships don't give a methodology to compute ignition delay for other types of fuels such as biodiesel. Most of the coefficients used in these relationships refer to experiments conducted in engine running under conventional diesel, thus leading one to the search of suited relationship which can take into account certain physical and chemical specificities of fuel used.

The present work, describes a semi empirical model used to predict how performances parameters such as thermal efficiency, specific fuel consumption, indicative pressure and indicative pressure will vary depending type of fuel used. The model is developed Fuel for diesel engine control purpose when running on biodiesel. Spray behavior of biodiesel is not covered in this work but some useful computational studies about the topic can be found in $[14,15]$, and it will be inserted in further development of the model.

\section{Governing Equations}

The model used in the present study is a semi-empirical model, based on the work of I. I. Wiebe [1] and the heat release calculation model of Watson [2] and the work of Grondin [3], Figure 1 shows a flow chart description of the model, where one can see the input information needed as well as the output data obtained from the model. The next section presents the main governing equations of the model as well as the constant used for our calculations. The model was implemented using an open source calculation code for rapid treatment of the information.

\subsection{Ignition Delay Model}

The initial Wiebe model was not taking into account the ignition delay period when calculating the combustion process. Wiebe model computes the heat release starting from the injection start angle, whereas it has been shown that ignition occurs after a certain amount of time after injection (ignition delay), due to complex chemistry processes.

In our model we used the model proposed by Hardenberg and Haze [4] which takes into account one of the main properties of the fuel which is believed to influence the delay period as in the cetane number.

$$
\begin{aligned}
I D= & {\left[0.36+0.22 \overline{U_{p}}\right] \exp \left[\frac { 6 1 8 8 4 0 } { C N + 2 5 } \cdot \left(\frac{1}{R T_{i m} \varepsilon^{n_{c}-1}}\right.\right.} \\
& \left.\left.-\frac{1}{17190}\right)+\left(\frac{21.2}{P_{i m} \varepsilon^{n_{c}}-12.4}\right)^{0.63}\right]
\end{aligned}
$$

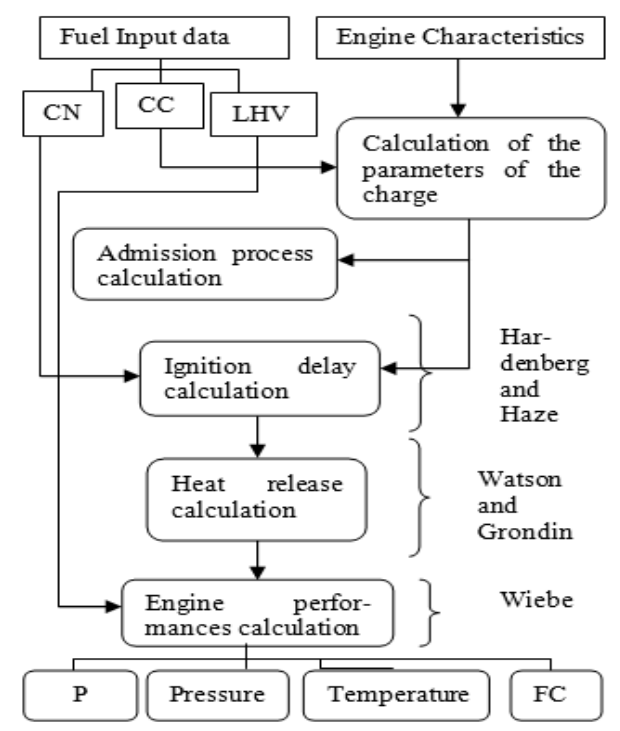

Figure 1. Model algorithm; $\mathrm{CN}$-Cetane number; CCChemical composition; P-Power; FC-Fuel consumption; LHV_Lower heating value. 
where $U_{P}$ is the mean piston speed in $\mathrm{m} / \mathrm{s} ; R$ is the gas constant in $\mathrm{J} / \mathrm{kmol}-\mathrm{K} ; \varepsilon$ is the compression ratio of the engine; $C N$ is the cetane number of the fuel $P_{i m}$ and $T_{i m}$ are the pressure in bar and temperature in Kelvin at the intake manifold; $n_{c}$ is the polytropic exponent for compression.

\subsection{Step by Step Computation}

The next step of the model is the fuel engine cycle model; here we determine the pressure inside the cylinder at any angle of rotation of the crankshaft taking into account the start of injection angle the duration of combustion and other parameters. As a result one will be able to determine the different parameters characterizing the efficiency of work of the engine, such as the specific consumption, the effective power and efficiency. The model computes the full process taking place inside the cylinder, with the calculations being made for each stroke of engine cycle.

\subsection{Admission Stroke}

The pressure of the working medium at the end of the admission stroke is given in Pascal by

$$
P_{a}=\frac{1}{\varepsilon}\left[(\varepsilon-1) \eta_{v} \cdot P_{k} \cdot \frac{T_{k}+\Delta T}{T_{k}}+P_{r}\right],
$$

where $\eta_{v}$ is the admission coefficient, $P_{k}$ is the pressure at inlet valves in $\mathrm{MPa}, T_{k}$ the temperature at inlet valves in $\mathrm{K}, \Delta T$ is the temperature gradient due to the heating of engine elements in $\mathrm{MPa}, P_{r}$ is the residual gases pressure in MPa.

The temperature of the working medium at the end of the admission stroke is given in Kelvin by

$$
T_{a}=\frac{T_{k}+\Delta T+\varsigma T_{r}}{1+\varsigma},
$$

where $T_{k}$ is the temperature of residual gases in $\mathrm{K}, \varsigma$ is the coefficient of residual gases.

The theoretically necessary (stoichiometric) quantity of air for the combustion of $1 \mathrm{~kg}$ of fuel is given as $L_{0}^{\prime}$, its value is dependent of the chemical composition of the fuel used and is determined by

$$
L_{0}^{\prime}=\frac{1}{0.23}\left(\frac{8}{3} C+8 H-O\right),
$$

where, $C, H$ and $O$ are respectively, the ratio of carbon, hydrogen and oxygen in the fuel chemical composition.

The specific volume of the working medium at the end of the admission stroke is given in $\mathrm{m}^{3} / \mathrm{kg}$ by

$$
v_{a}=0.008314 \frac{T_{a}}{\mu_{\text {air }} P_{a}},
$$

where $\mu_{\text {air }}$ is the molecular mass of air.

\subsection{Compression Stroke}

The parameters of the working medium during the compression stroke are computed using the polytropic process equation.

The pressure at a given time is given in MPa by

$$
P=P_{a}\left(\frac{v_{a}}{v}\right)^{n_{c}}
$$

where $v$ is the current value of specific volume defined as

$$
v=\left(\frac{v_{a}}{\varepsilon}\right)\left(1+\frac{\varepsilon-1}{2} \sigma\right),
$$

$\sigma$ is the kinematic function of the motion of the piston

$$
\sigma=1-\cos \varphi+\frac{1}{\lambda}(1-\cos (\arcsin (\lambda \sin \varphi))),
$$

where $\lambda$ is the ratio of the lengths of the crankshaft and the connecting rod, $\varphi$ is the current angle of rotation of the crankshaft.

The specific work of compression is then determined in $\mathrm{MJ} / \mathrm{Kg}$ by

$$
l_{a y}=\frac{\left(P_{a} v_{a}-P_{y} v_{y}\right)}{n_{c}-1}
$$

\subsection{Power Stroke}

\subsubsection{Heat Release Model}

The admission, compression and ignition delay phase being computed, the next is step of the model is the heat release calculation. Our heat release model computes two phases of the combustion process, the premixed and diffusion phase.

The current fraction of fuel burnt $x=f(\varphi)$-where $\varphi$ is the current crankshaft position angle-is computed using a double Wiebe function [2], we then have

$$
x=\beta x_{p}+(1-\beta) x_{d},
$$

with $x_{p}$ and $x_{d}$ representing the fraction of fuel burnt in each phase of the combustion process, $\beta$ representing the fraction of fuel injected during the premixed phase.

For each phase of the combustion phase (Equation 10) we can write

$$
x_{i}=1-\exp \left[-a_{i}\left(\frac{\varphi-\varphi_{\text {comb }}}{\Delta \varphi_{i}}\right)^{m_{i}+1}\right],
$$

where $a i$ and $m_{i}$ are experimental shaping coefficient of the Wiebe function; $\varphi_{\text {comb }}$ is the start of ignition angle; $\Delta \varphi_{i}$ is the combustion duration for each phase.

The normalized combustion rate $(1 / \mathrm{deg})$ is computed by derivation of $x_{i}$ about $\varphi$. 


\subsubsection{Combustion Model}

The combustion effectiveness which accounts for heat loss (heat loss due to heat transfer to the walls, hydraulic losses due to the flow of gases) ratio is first defined by

$$
\xi=\psi \delta
$$

where $\delta$ is a heat release factor which takes into account the ration of unburned fuel; $\psi$ - is a ratio of used heat.

The total specific heat of combustion used (instantaneous heat release) is given in $\mathrm{MJ} / \mathrm{Kg}$ by

$$
q_{z}=\frac{\xi H_{u}}{(1+\gamma) \alpha L_{0}^{\prime}+1},
$$

where $H_{u}$ is the net calorific value of the used fuel in $\mathrm{MJ} / \mathrm{Kg}$ and $\alpha$ is the coefficient of excess air and $\gamma$ is the ratio of specific heat during the combustion process, according to [16] is value can be taken as constant going from 1.3 to 1.35 .

Pressure calculation at any moment of the power stroke is computed using the first law of thermodynamic and can described for the evolution of the pressure/volume indicator diagram from a point 1 to 2 by

$$
q_{1-2}=C_{v 1-2}\left(T_{2}-T_{1}\right)+\int_{v_{1}}^{v_{2}} p \mathrm{~d} v,
$$

where: $q_{1-2}$ specific heat used to increase the internal energy from point 1 to 2 in the diagram; $C_{v 1-2}$ is the average specific heat the working medium for constant pressure from point 1 to 2 .

Assuming each volume step is small enough and using the trapezoidal method to simplify the integral in formula (14) and expressing $C_{v 1-2}$ in term of $P_{2}$ using the Mayer's formula, we determine the value of the pressure at any given time of the power stroke using the simplified equation

$$
p_{2}=\frac{2 q_{z} \Delta x_{1-2}+p_{1}\left(\left(\frac{\gamma+1}{\gamma-1}\right) \cdot v_{1}-v_{2}\right)}{\left(\frac{\gamma+1}{\gamma-1}\right) \cdot v_{2}-v_{1}},
$$

$\Delta x_{1-2}$ is the ratio of fuel burnt from point 1 to 2 .

Specific work of gases during the combustion stroke is given in $\mathrm{MJ} / \mathrm{Kg}$ by

$$
L_{y z}=\sum_{1}^{n} \frac{p_{1}+p_{2}}{2}\left(v_{2}-v_{1}\right) .
$$

Further description of the model can be found in [9], here we presented the main equations for combustion that will have a greater impact on the engine performance calculation.

\section{Results and Discussion}

\subsection{Model Validation}

The validation of the model consisted in comparing ex- perimental results from previous researches with simulation results using the model. For this purpose, experimental data obtained by Sahoo et al. [17] from a single cylinder diesel at rated speed were compared with the model simulation. To further investigate the validity of the model we proceeded to the simulation of a six cylinder direct injection diesel at different crankshaft rotational speed, results were compared with experimental data obtained by Ahmet et al. [18].

The simulation were performed on a 3 Go of RAM Dual core computer with a time step of 0.1 crank angle degrees, a full simulation took about 6 minutes to complete. The parameters of the model were adjusted to closely match the experimental results. The diffusive combustion duration was estimated about 75 and 60 degrees of rotation of the crankshaft for the first and second experiment respectively using least square fitting technique.

\subsubsection{Experimental Setup}

Tables 1 and 2 present the engines specifications for each experiment. The default injection timing for the second experiment was determined from the fuel line pressure diagram. The second experiment was performed under varying crankshaft rotational speed-1000, 1250, 1500,1750 and $2000 \mathrm{rpm}$.

The calorific value of the diesel fuel was also modified for the second experiment to $42.5 \mathrm{MJ} / \mathrm{kg}$ to match experimental input data, remaining properties of the fuel were kept unchanged. To perform comparison we used fuel characteristics that were stated in the work of Sahoo et al. [17], but due to the lack of some relevant information that shall be given as entry parameters to our model we had to add them according to diesel fuel standards. Specifications of the validating fuel can be seen at Table 3.

\subsubsection{Comparison of Experimental result with Simulation}

Figure 2 shows the in cylinder pressure during the combustion phase for experiment number 1. It can be seen that the simulated curve has a similar shape with the experimental curve, the peak pressure, starting and ending pressure values are almost the same. The slight gap between the curves can be due to the fact that the ignition delay model underestimated the experimental ignition delay obtained.

Table 4 shows comparison of simulation results to experimental performances results. It can be seen than the peak pressure is estimated with good accuracy by the model. However the occurrence of the peak pressure is about six degrees earlier in the simulated result, this is an issue that should investigated in further researches. The break power is estimated with an accuracy of $95 \%$, 
Table 1. First experiment engine specifications.

\begin{tabular}{ccc}
\hline No. & Particular & Specifications \\
\hline 1 & Make & Kirloskar Oil engine \\
2 & Model & DAF 8 \\
3 & Rated brake power $(\mathrm{kW})$ & 6 \\
4 & Rated speed (rpm) & 1500 \\
5 & Number of cylinder & 1 \\
6 & Bore $\times$ Stroke $(\mathrm{mm})$ & $95 \times 110$ \\
7 & Compression ratio & $17.5: 1$ \\
8 & Fuel injection timing & $23^{\circ}$ \\
\hline
\end{tabular}

Table 2. Second experiment engine specifications.

\begin{tabular}{ccc}
\hline No. & Particular & Specifications \\
\hline 1 & Make & Ford \\
2 & Model & 6.0 Ford Cargo \\
3 & Rated brake power $(\mathrm{kW})$ & 81 \\
4 & Rated speed (rpm) & 2600 \\
5 & Number of cylinder & 6 \\
6 & Bore $\times$ Stroke $(\mathrm{mm})$ & $104.8 \times 114.9$ \\
7 & Compression ratio & $15.9: 1$ \\
8 & Fuel injection timing & $20^{\circ}$ \\
\hline
\end{tabular}

Table 3. Fuel specification used for model validation.

\begin{tabular}{ccc}
\hline No. & Particular & Specifications \\
\hline 1 & Make & Diesel \\
2 & Calorific value & $44 \mathrm{MJ} / \mathrm{kg}$ \\
3 & Carbon content & $87 \%$ \\
4 & Hydrogen content & $12.6 \%$ \\
5 & Oxygen content & $0.4 \%$ \\
6 & Density $\left(\mathrm{kg} / \mathrm{m}^{3}\right)$ & 850 \\
7 & Viscosity at $40^{\circ} \mathrm{C}(\mathrm{Cst})$ & 2.87 \\
8 & Cetane number & 51 \\
\hline
\end{tabular}

Table 4. First experiment comparison with simulation results.

\begin{tabular}{ccc}
\hline & Experimental results & Simulation results \\
\hline Ignition delay & 8.5 & 6.88 \\
Start of combustion BTDC & 14.5 & 16.12 \\
In cylinder maximum & 78.7 & 79.412 \\
pressure (bar) & & \\
Peak pressure occurrence & $5.8 \mathrm{ATDC}$ & $2.6 \mathrm{BTDC}$ \\
Brake power & 6 & 5.75 \\
\hline
\end{tabular}

which is fairly acceptable. This proves that despite the slight gap observed our model can be used to estimate the overall performances for this particular engine.

The second experiment possessed a wider scope because it permitted to evaluate the model in a broader aspect. The compared experiment consisted in determining the engine performance at various crankshaft rotational speeds. Table 5 shows the comparisons of engine per-

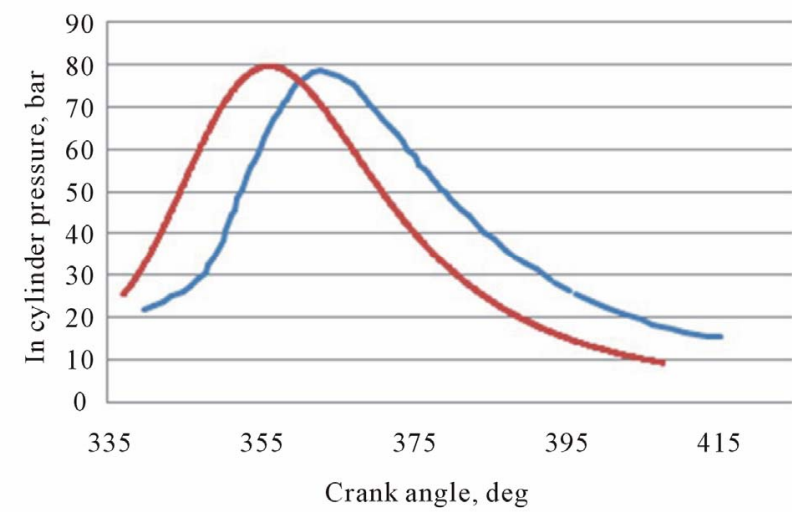

Figure 2. Experimental - and - simulated in cylinder pressure during combustion phase in the first experiment.

Table 5. Second experiment comparison with simulation results.

\begin{tabular}{|c|c|c|c|c|c|c|}
\hline \multirow[b]{2}{*}{ RPM } & \multicolumn{2}{|r|}{$\begin{array}{l}\text { Brake } \\
\text { power }\end{array}$} & $\begin{array}{l}\text { Maximum in } \\
\text { cylinder } \\
\text { pressure, MPa }\end{array}$ & \multicolumn{3}{|c|}{$\begin{array}{l}\text { Maximum in cylinder } \\
\text { pressure } \\
\text { occurence, CA ATDC }\end{array}$} \\
\hline & $\mathbf{E}$ & $\mathrm{S}$ & $\mathbf{E}$ & $\mathrm{S}$ & $\mathbf{E}$ & $\mathbf{S}$ \\
\hline 1000 & 32.3 & 33.38 & 8.04 & 7.89 & 5.3 & 5.8 \\
\hline 1250 & 42.3 & 41.30 & 8.6 & 7.92 & 6.5 & 5.8 \\
\hline 1500 & 52.5 & 48.83 & 7.95 & 7.85 & 6.2 & 6 \\
\hline 1750 & 60.4 & 55.92 & 8.1 & 7.71 & 8.2 & 6.3 \\
\hline \multirow[t]{2}{*}{2000} & 68.3 & 62.56 & 8.04 & 7.56 & 7.4 & 6.4 \\
\hline & & \multicolumn{2}{|c|}{ BSFC, g/kWh } & \multicolumn{3}{|c|}{ Ignition delay, CA } \\
\hline RPM & & $\mathbf{E}$ & $\mathrm{S}$ & $\mathbf{E}$ & & $\mathbf{S}$ \\
\hline 1000 & & 269.4 & 209.64 & 8.15 & & 7.27 \\
\hline 1250 & & 265.104 & 211.84 & 8.15 & & 7.59 \\
\hline 1500 & & 254.6 & 215.01 & 8.15 & & 8.09 \\
\hline 1750 & & 267.9 & 219.03 & 8.15 & & 8.68 \\
\hline 2000 & & 274.175 & 223.75 & 8.15 & & 9.32 \\
\hline
\end{tabular}

CA — Crank angle, ATDC-After top dead center, E-Experiment, SSimulation, BSFC - Break specific fuel consumption.

formances obtained from the experiment with those obtained using the present model.

As observed for the first experiment, the model predicts with good accuracy the power output and peak in cylinder pressure of the engine independently of crankshaft rotational speed.

The break specific fuel consumption is estimated with an accuracy of about $18 \%$, this can be explained by the fact that the model doesn't take into account in a deeper aspect, the fuel injection process, the fuel flow, hydraulic losses, injector geometry and number. These parameters shall be investigated in future researches to improve the accuracy of the model.

The average simulated ignition delay matched the experimental result contrary to the result obtained in the first experiment. This lead to a reduction of the gap observed in the first simulation. It can be seen in Figure 3 


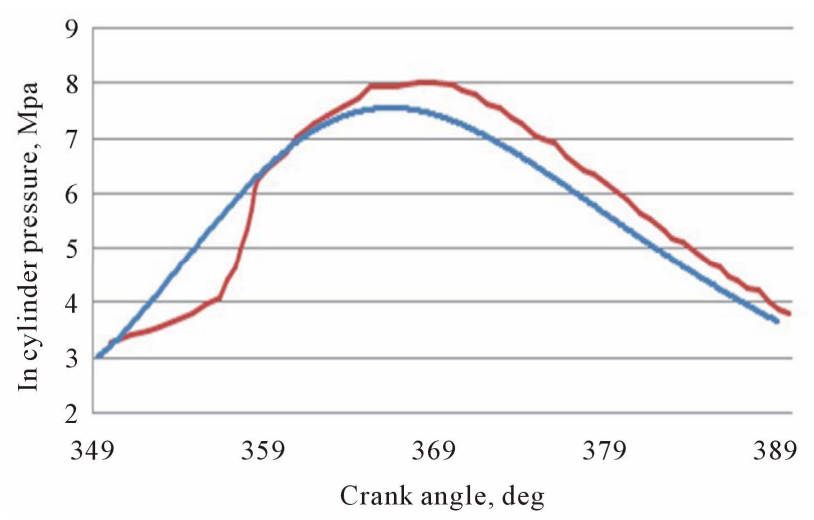

Figure 3. Experimental - and simulated - in cylinder pressure during combustion phase in the second experiment at $2000 \mathrm{rpm}$.

that the in cylinder curves better matched compared to the first experiment. This can be explained by the fact ignition delay models are often dependent of the experimental setup in which they were determined [4], thus giving slightly different results depending on the context where they are used. The motivation of the selection of our ignition delay model has been discussed above and the good accuracy of our results was encouraging.

These results show that the model predicts fairly well the overall engine performances of the investigated engines. It is worth noting that the model uses many experimental constant taken from $[9,10,19,20]$. The selection of these constants should be accurate to improve the accuracy of the model.

Most of the parameters of the model have to be adjusted in order to obtain a better fit with experimental results. A better use of the present model in engine setting-up and diagnostic situations can be achieved with preliminary experimental investigation on the specific engine investigated.

The model having been validated with relevant experimental results, and shown it can be used for engine performances predictions, it was further used for the evaluation of a direct injection engine fuelled by biodiesel fuel. This study follows in the next section.

\subsubsection{Simulation of a DI Diesel Engine Performance Fuelled on Biodiesel}

The final part of this study was the simulation of a DI diesel engine performance fuelled by palm oil biodiesel fuel. The properties of the biodiesel fuel are taken from [3] and are shown on Table 6.

Simulations were performed for unchanged engine parameters used for the first model validation. Result showed that peak cylinder was higher for biodiesel fuel as well as in cylinder temperature (Figures 4 and 5). This confirms results obtained from $[3,17,21]$. This can be due to the higher content of oxygen in biodiesel fuel that tends
Table 6. Biodiesel fuel specification.

\begin{tabular}{ccc}
\hline No. & Particular & Specifications \\
\hline 1 & Make & Biodiesel \\
2 & Calorific value & $40 \mathrm{MJ} / \mathrm{kg}$ \\
3 & Carbon content & $76.5 \%$ \\
4 & Hydrogen content & $12.3 \%$ \\
5 & Oxygen content & $11.1 \%$ \\
6 & Density $\left(\mathrm{kg} / \mathrm{m}^{3}\right)$ & 883 \\
7 & Viscosity at $40^{\circ} \mathrm{C}(\mathrm{Cst})$ & 6.83 \\
8 & Cetane number & 52 \\
\hline
\end{tabular}

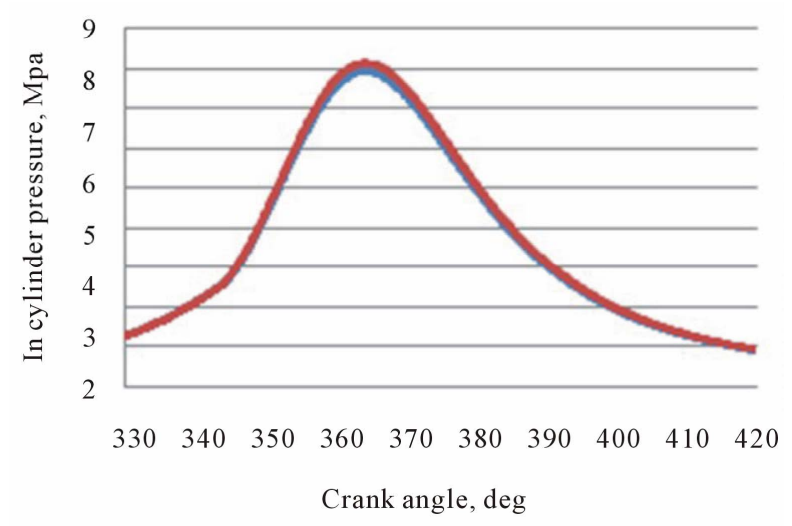

Figure 4. In cylinder pressure for diesel - and biodiesel - at nominal rpm.

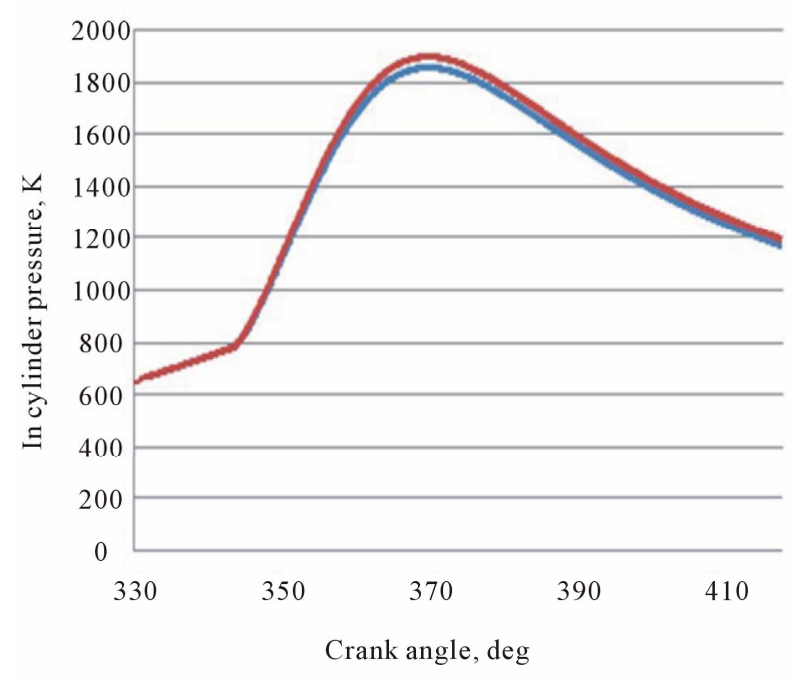

Figure 5. In cylinder temperature for diesel - and biodiesel - at nominal rpm.

to produce a higher heat release rate in the cylinder and a better burning rate.

Table 7 shows comparatives results of the engine performance when running on biodiesel and diesel fuel. The ignition delays value for both fuel are almost equal, this is due to their cetane numbers which are about the same value. Break powers obtained are also very close 
Table 7. Comparison of simulated engine performance using biodiesel and diesel as fuel.

\begin{tabular}{ccc}
\hline & Biodiesel & Diesel \\
\hline Ignition delay & 6.82 & 6.88 \\
In cylinder maximum pressure (bar) & 81.392 & 79.412 \\
Break thermal efficiency & $48.4 \%$ & $48.0 \%$ \\
Brake power & 5.748 & 5.747 \\
Break specific fuel consumption $(\mathrm{g} / \mathrm{kWh})$ & 185.73494 & 170.27237 \\
\hline
\end{tabular}

with the biodiesel giving a slightly higher value. The main difference comes when analyzing the break specific fuel consumption (BSFC); it can be seen that BSFC is $10 \%$ higher for biodiesel than for conventional diesel. This is due to the lower value of the net calorific value of the biodiesel fuel. That means for equal power output one needs to spend more on fuel when using biodiesel as fuel for diesel.

Overall, the model is predicting well the trends observed in different previous researches, its further development is expected to provide an engine analysis tool for simulation, conception and diagnostic.

\section{Conclusions}

A semi empirical 0D model was elaborated to predict diesel engine performance when running on biodiesel. The model was first validated with experimental results from a single cylinder engine then simulations were made to analyze the engine performance when running on biodiesel compared to the performances obtained with diesel fuel. Final results of the study showed that, the model matches the overall experimental data, such as the power output and peak cylinder pressure. The simulations of engine performance when running on biodiesel were in good accordance with results obtained in previous experimental researches on biodiesel.

The ignition delay was underestimated by the model for the first experiment and well predicted for the second. A slight gap in cylinder pressure curve was observed for both experimental comparison but it didn't affect the overall engine performances prediction. The model can be extended to a "decision making" tool for diesel engine setup and diagnostic.

Further researches will be made to better the accuracy of the model and to account for fuel injection study. The model will be further investigated for engine control when shifting to biodiesel fuel.

\section{Acknowledgements}

The authors have greatly benefited from discussion and comments with Professor Manaswita BOSE from the Indian Institute of Technology of Bombay on the present research.

\section{REFERENCES}

[1] D. Ayhan, "Progress and Recent Trends in Biofuels," Progress in Energy and Combustion Science, Elsevier, 2007, pp. 1-18.

[2] M. Lapuerta, O. Armas and J. R. Fernandez, "Effect of Biodiesel Fuels on Diesel Engine Emissions," Progress in Energy and Combustion Science, Elsevier, 2008, pp. 198223.

[3] A. P. Sathiyagnanam and C. G. Saravanan. "Experimental Studies on the Combustion of a Direct Injection Engine Fueled with SCR," Proceedings of the World Congress on Engineering, 6-8 July 2011, London.

[4] P. A. Lakshminarayanan and Y. V. Aghav, "Modelling Diesel Combustion,” Springer, 2009.

[5] Y. Shi, H.-W. Ge and R. D. Reitz, "Computational Optimization of Internal Combustion Engines," Springer, 2011. http://dx.doi.org/10.1007/978-0-85729-619-1

[6] Technologies Gamma, "Gamma Technologies-Engine and Vehicle Simulation," 2013. http://www.gtisoft.com/

[7] P. L. C. Ricardo, "Home-Ricardo," 2013. http://www.ricardo.com/en-GB/

[8] S.A.S Scilab Enterprises, "Home-Scilab," Scilab Enterprises S.A.S, 2013. http://www.scilab.org/en

[9] I. I. Wiebe, "Novelty on Working Process of Internal Combustion Engine (Speed of Combustion and Working Process in Engine) (In Russian)," MachGiz, Sverdlovsk, 1962.

[10] G. Olivier, "Modélisation du Moteur à Allumage par Compression dans la Perspective du Contrôle et du Diagnostic (PhD Thesis)," Université de Rouen, Rouen, 2004.

[11] F. Pischinger, E. Scheid and U. Reuter, "Influences of Fuel Quality and Injection Parameters on Self Ignition of Sprays," Warsaw, 1987.

[12] N. F. Razleytsev, "Combustion Simulation and Optimization in Diesels (In Russian)," Vischa Shkola, Kharkov, 1980, p. 169.

[13] A. S. Lyshevsky, "Fuel Atomization in Marine Diesels (In Russian)," Leningrad, 1971.

[14] X. Wang, K. Li and W. H. Su, "Experimental and Numerical Investigations on Internal Flow Characteristics of Diesel Nozzle under Real Fuel Injection Conditions," Experimental Thermal and Fluid Science, Elsevier, 2012, pp. 204-211.

[15] X. G. Wang, Z. H. Huang, O. A. Kuti, W. Zhang and K. Nishida, "An Experimental Investigation on Spray, Ignition and Combustion Characteristics of Biodiesels," Proceedings of the Combustion Institute, Elsevier, 2011, pp. 2071-2077.

[16] J. Heywood, "Internal Combustion Engine Fundamentals," McGraw-Hill Series in Mechanical Engineering, 1988, pp. 560-561.

[17] P. K. Sahoo and L. M. Das, "Combustion Analysis of Jatropha, Karanja and Polanga Based Biodiesel as Fuel in a Diesel Engine," Fuel, Elsevier, 2009, pp. 994-999.

[18] A. N. Ozsensen and M. Canakci, "Determination of Per- 
formance and Combustion Characteristics of a Dieselengine Fueled with Canola and Waste Palm Oil Methyl Esters," Energy Conversion and Management, Elsevier, 2011, pp. 108-116.

[19] N. Watson, D. A. Pilley and M. Marzouk, "A Combustion Correlation for Diesel Engine Simulation," Society of American Engineers, 1980. http://dx.doi.org/10.4271/800029

[20] F. W. Hardenberg and H. O. Hase, "An Empirical Formula for Computing the Pressure Rise Delay of a Fuel from Its Cetane Number and from the Relevant Parameters of Direct Injection Diesel Engines," Society of Automotive Engineers, Warrendale, 1979.

[21] B. Tesfa, R. Mishra, F. Gu and A. D. Ball, "Combustion Characteristics of CI Engine Running with Biodiesel Blends," Environment and Power Quality European Association for the Development of Renewable Energies, International Conference on Renewable Energies and Power Quality, Las Palmas de Gran Canaria, 2011. 